Volume 8, No. 7, July - August 2017

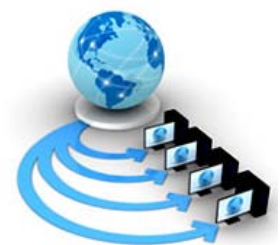

International Journal of Advanced Research in Computer Science

RESEARCH PAPER

Available Online at www.ijarcs.info

\title{
A COMPARATIVE STUDY ON LEACH AND RE REAC-IN PROTOCOL IN WIRELESS SENSING NETWORK
}

\author{
Mandeep Sidhu \\ Department of E.C.E. \\ B.G.I.E.T. \\ Sangrur (Punjab), India
}

\author{
Manpreet Singh \\ Department OF E.C.E. \\ B.G.I.E.T. \\ Sangrur (Punjab), India
}

\begin{abstract}
ABSTACT: wireless sensor networks consists of sensor nodes which are powered by battery, to communicate with each other for various operations, energy efficiency is the main issue in wireless sensor networks. Therefore, to maximize network lifetime and maximum reliable and scalability routing techniques have been developed LEACH is the conventional hieriacial clustering protocol widely used in wsn , RE REAC-IN is protocol used on residual energy for clustering. In this paper we will compare LEACH protocol along with RE REAC -IN protocol with its advantages and disadvantages .the objective of this paper is to provide comparison results between LEACH and RE REAC -IN protocols.
\end{abstract}

Keywords: Wireless sensing network (wsn) ;LEACH (low energy adaptive clustering hierarchy routing protocols) ;

Re REAC-IN (residual Regional aware clustering with isolated nodes; clustering; packet delivery ratio ; uncovered nodes ; throughput ; remaining energy ; energy efficiency .

\section{INTRODUCTION}

Wireless Sensor Networks(WSN) have increased overall consideration as of late because of the advances made in remote correspondence, data advances and hardware field. The idea of wireless sensor systems depends on a straightforward condition: Sensing $+\mathrm{CPU}+$ Radio $=$ Thousands of potential applications.

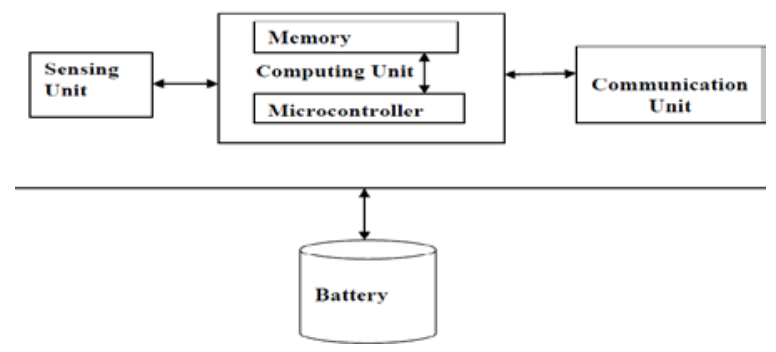

Fig 1.1: basic structure of wsn

It is a detecting innovation where small, self-governing and minimal gadgets called sensor nodes sent in a remote territory to recognize events, gather and process information and transmit detected data to clients. The advancement of minimal effort, low-control, a multifunctional sensor has gotten expanding consideration from different commercial ventures. Sensor nodes in WSNs are little estimated and are fit for detecting, assembling and handling information while speaking with other associated nodes in the system, by means of radio. WSN term can be extensively detected as gadgets reach from portable workstations, PDAs or cell telephones to extremely little and basic detecting gadgets. At present, most accessible remote sensor gadgets are significantly obliged as far as computational force, memory, productivity and correspondence abilities because of financial and innovation reasons. That is the reason the vast majority of the exploration on WSNs has focused on the configuration of vitality and computationally productive calculations and conventions, and the application space has been bound to basic information situated checking and reporting applications. In the event that WSNs nodes are all the more capable or mains-fueled gadgets in the region, it is helpful to use their calculation and correspondence assets for complex calculations and as doors to different systems [1] Clustering sensor nodes is a successful topology control technique to reduce energy consumption of the sensor nodes for maximizing lifetime of WSNs. Clustering the nodes has many advantages, such as scalability, energy efficiency and reducing routing delay. Cluster based routing involves the creation of clusters, election of cluster heads and routing through the Cluster Heads $(\mathrm{CH})$. The energy can be conserved more by the $\mathrm{CH}$ by collecting the data in the cluster, compressing it and then transmitting the aggregated data to the base station. The two imperative steps in clustering scheme are $\mathrm{CH}$ determination and cluster formation. Improperly designed clustering algorithms can cause nodes to become isolated from $\mathrm{CHs}$ as shown in Fig. 1.2 Communication of these isolated nodes with the sink will consume more energy. If the sink is far away from these isolated nodes, then isolated nodes cannot directly communicate with the sink. If these isolated nodes are not in the range of nearby current cluster heads, then isolated nodes cannot communicate with the current cluster head also. This paper presents the brief study about past researches that have been by various authors in Section II. Further, Section III represents the proposed work and finally section IV and V represents results and conclusion part. 


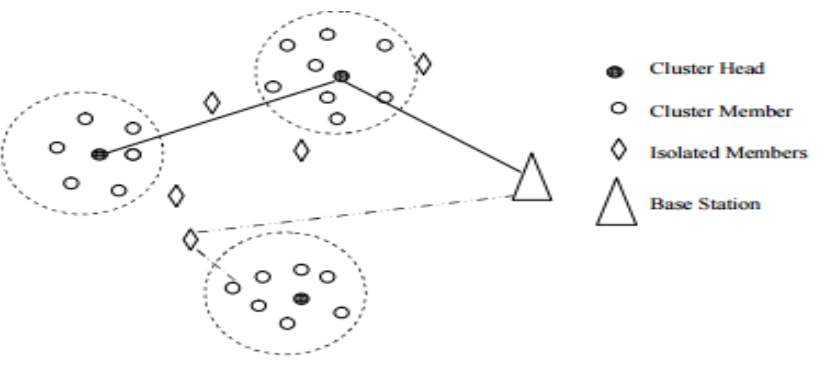

Figure 1.2 Wireless sensor network with isolated nodes [2]

\section{EXISTING WORK}

Mandeep Sidhu and Manpreet Singh [1] The nodes in wireless sensor networks are usually deployed in the areas where human approach is very difficult. In such cases, the deployment of the nodes tends to be random from topological point of view. The main thing that needs to be given importance in such kind of networks is their lifetime which tends to be very less as the nodes are battery operated nodes. So if any of the sensors dies out because of energy drainage it is very difficult to come up with their replacement. The replacement of the drained out sensor nodes tends to become a costly affair. In such scenarios the focus must be given on reducing the energy consumption of the sensor nodes so that they can transfer the data over a longer period of time. The solution to this is clustering of the nodes in groups based on some rules that would eventually increase their working time in the network. The rules laid out for clustering is usually defined in LEACH protocol that tends to form the clusters and select their leader's randomly using threshold criterion. However, the clustering of the network results should be optimal enough such that all the nodes are included in the cluster. In previous paper they explores the clustering protocol and aims to optimize the clustering process to improve the lifetime of the network. The proposed scheme has shown better performance in terms of energy consumption, packet delivery ratio and throughput.

Jenq-ShiouLeu.et.al. [4] A reasonable clustering algorithm for gathering sensor nodes can build the vitality productivity of WSNs. Notwithstanding, clustering requires extra overhead, for example, cluster head choice and task, and cluster development. This paper proposes another provincial vitality mindful clustering technique utilizing detached nodes for WSNs, called Regional Energy Aware Clustering with Isolated Nodes (REAC-IN). In REAC-IN, CHs are chosen in view of weight. Weight is resolved by leftover vitality of every sensor and the territorial normal vitality of all sensors in every cluster. Dishonorably planned circulated clustering algorithms can make nodes get to be detached from CHs. Such detached nodes speak with the sink by devouring abundance measure of vitality. To drag out system lifetime, the provincial normal vitality and the separation amongst sensors and the sink are utilized to figure out if the detached node sends its information to a $\mathrm{CH}$ node in the past round or to the sink. A WSN is a blend of remote correspondence and sensor nodes. The system must be vitality proficient and stable, and have a long lifetime. The REAC-IN convention displayed in this paper enhances the cluster head determination handle and takes care of the issue of node isolation. The outcomes uncovered that the execution of the algorithms utilized as a part of REACIN to enhance the lifetime and dependability of a system is more good than that of the algorithms utilized as a part of different conventions.

M.Natarajan 2013 [5] proposed proper organization of nodes becomes one of the major techniques to expand the life span of the whole network through aggregating data at the cluster head $(\mathrm{CH})$. LEACH (Low Energy Adaptive Clustering Hierarchy) and PSO (Particle Swarm Optimization) are applied for producing energy-aware clusters with optimal selection of cluster head. The determination of a group head utilizing PSO minimizes the intra cluster separation between group head and the cluster part, and the improvement of vitality administration of the system. From the recreation results, it is seen that Vitality Mindful Optimal cluster head choice utilizing PSO approach builds the system lifetime of the group in such a route by diminishing the aggregate vitality utilization than Drain execution.

D. J. Dechene. et. al. [7] in this paper, they inspect at present proposed clustering algorithms for Wireless Sensor Networks. They examine the operations of these algorithms, and also draw correlations on the execution between the different plans. The present state of proposed clustering conventions, particularly as for their energy and unwavering quality necessities. In remote sensor systems, the vitality confinements of nodes assume a significant part in outlining any convention for execution. Likewise, Nature of Service measurements, for example, delay, information misfortune resistance, what's more, system lifetime uncovers dependability issues when outlining recuperation systems for clustering plans.

Xia Li et al., 2013 [8] proposed technique in which they join the enhanced molecule swarm grouping calculation with the between bunch directing calculation to shape a versatile vitality proficient grouping directing convention, alluded to as AECRP. Reenactment results demonstrate that this convention not just adjusts the vitality utilization of the general system, postpones the demise time of the nodes, additionally gives more dependable information conveyance.

Agam et al., 2014 [8] proposed the self-knowledge technique in which SCHP (sub cluster head protocol is used to reduce delay occurs in link stability problem in SCHP. Each node knows about its neighbors by using its selfknowledge. Receiver will send its receiving capacity and sender will send data according to that capacity. So link becomes more stable. In Self-knowledge technique sender keeps the information regarding its one hop neighbors. Allimportant parameters like delay, threshold, packet size, energy are considered before sending data to the next node. A minimum criterion is set for all the parameters, among all the neighboring nodes the nodes satisfying this minimum criterion are considered for the candidates of relay node. Among these nodes the node with lowest delay and maximum energy is chosen as relay node. If this node dies after sometime then the next eligible candidate is chosen as relay node and attempt is made to reconfigure the dead node. This technique provides a better link stability than simple SCHP by avoiding congestion in the link because the sender sends the data according to the receiving capacity of 
the receiver. So the time and energy wasted in reestablishing the link that can be lost due to congestion is saved that accounts in increasing the lifetime and reducing the delay.

SK Kajal Arefin Imon et. al. [11] the creator has proposed a tree structure established at the sink is characterized. Contingent upon different components, including the WSN topology and the accessibility of assets, the vitality utilization of nodes in various ways of the information accumulation tree may differ to a great extent, in this way influencing the general system lifetime. This paper addresses the issue of lifetime amplification of WSNs in view of information accumulation trees. we propose a novel furthermore, proficient algorithm, called Randomized Switching for Augmenting Lifetime (RaSMaLai), that goes for developing the lifetime of WSNs through load adjusting. Given an introductory information accumulation tree, RaSMaLai arbitrarily switches some sensor nodes from their unique ways to different ways with lower stack. We demonstrate that, under fitting settings of the working parameters, RaSMaLai joins with a low time many-sided quality. In light of the idea of limited adjusted trees, our algorithm arbitrarily switches the information sending ways of nodes. We have given a basic yet compelling exchanging procedure that outcome in a quick merging. We have additionally introduced a disseminate usage of our plan that has a low overhead. A broad study through both recreations and investigations on a genuine WSN test affirmed that our approach can altogether increment the system lifetime with a lower time intricacy than the present cutting edge in a wide range of working conditions.

Heena dhawan and sandeep wariach [6] proposed various types of LEACH protocols and their working on comparison basis.

\section{PROPOSED WORK}

\section{Comparison between CLUSTERED-BASED HIERARCHICAL ROUTING PROTOCOLS:}

\subsection{Low-Energy Adaptive Clustering Hierarchy} (LEACH)[1][4][6], is a typical hierarchical clustering routing protocol, which adopts distributed clustering algorithm where cluster-head rotation mechanism, data aggregation, and data fusion technologies effectively improves the lifetime of network. In order to optimize energy in the network, nodes are selected as cluster head circularly and randomly. The normal nodes called cluster members join the corresponding cluster head nodes on the basis of principle of proximity. Normal nodes sense data and send directly to the cluster head nodes. The cluster head nodes receive sensed data, aggregate the data to remove redundancy and fusion processes are carried out and data is send to the sink (or Base Station). So LEACH increases network lifetime by decreasing network energy consumption, and reducing number of communication messages by data aggregation and fusion. Running Process of LEACH The Leach operation is classified into different rounds, and each of these rounds has mainly two phases: the Set-up Phase and the Steady-state for data transmission.

3.1.1 The Set-up Phase[1][4][6]: First, the LEACH protocol randomly selects cluster heads (CHs) by randomly generating a number $(\mathrm{n})$ between 0 and 1 for each node. If this randomly generated number is less than the threshold value given by threshold function $\mathrm{T}(\mathrm{n})$, the node would be selected as cluster head node. Where $\mathrm{P}$ is the cluster-head probability and $G$ is the set of nodes that never be chosen as cluster-head nodes before 1/p round . After the selection of cluster head nodes, each cluster-head node will send information via CDMA code to other nodes and normal nodes will join the corresponding cluster-head nodes. Then the cluster head nodes use TDMA to provide data transmission time for every node connected to them

3.1.2The Steady-state [1][4][6]: This stage is for data transmission where normal nodes sense data and send this sensed data to their respective cluster-head nodes. The processing of received data (data aggregation and data fusion) is done by cluster head

\section{DEFICIENCIES IN CLASSICAL LEACH PROTOCOL [6]}

4.1. Unreasonable cluster head selection: LEACH protocol doesn't take residual energy of each node into consideration for the selection of cluster head node as each node has equal probability of becoming cluster head. If low-energy node is being selected as cluster head node, then the network fails soon due to high energy consumption causes adverse to energy balancing among the network. This results data loss and lower in survival time of the network.

4.2. Unreasonable distribution of cluster heads: The random selection algorithm of cluster head nodes causes problem of imbalance in energy load. Distance factor is not considered in cluster formation due to which sometimes very big clusters and very class clusters exist at the same time in the network. More the distance between cluster head node and base station, more the energy consumption of that node.

4.3 More responsibility on Cluster Head node: Cluster head nodes perform data aggregation and send processed data to the base station in single-hop due to which cluster head nodes deplete their energy too fast as compared to normal nodes. Also if a cluster head node fails, the whole nodes linked to it will deplete their energy to nodes and processed data will be sent to the base station.

\section{RE REAC-IN PROTOCOL[1][4]}

A reasonable clustering algorithm for gathering sensor nodes can build the vitality productivity of WSNs. Notwithstanding, clustering requires extra overhead, for example, cluster head choice and task, and cluster development. Here another clustering technique utilizing detached nodes for WSNs, called Regional Energy Aware Clustering with Isolated Nodes (REAC-IN). In REAC-IN, $\mathrm{CHs}$ are chosen in view of weight. Weight is resolved by leftover vitality of every sensor and the territorial normal vitality of all sensors in every cluster. Dishonorably planned circulated clustering algorithms can make nodes get to be detached from CHs. Such detached nodes speak with the sink by devouring abundance measure of vitality. To drag out system lifetime, the provincial normal vitality and the separation amongst sensors and the sink are utilized to figure out if the detached node sends its information to a $\mathrm{CH}$ 
node in the past round or to the sink. A WSN is a blend of remote correspondence and sensor nodes. The system must be vitality proficient and stable, and have a long lifetime. The REAC-IN convention displayed in this paper enhances the cluster head determination handle and takes care of the issue of node isolation. The outcomes uncovered that the execution of the algorithms utilized as a part of REACIN to enhance the lifetime and dependability of a system is more good than that of the algorithms utilized as a part of different conventions.

\section{RESULTS}

The whole scheme has been implemented in NS2.35. The parameters used for simulation has been shown in the table below:

Table: Simulation Parameters

\begin{tabular}{|l|l|}
\hline Parameter & Value \\
\hline Channel & Wireless \\
\hline Propagation Model & Two Ray Ground \\
\hline Mac & 802.11 \\
\hline Number of nodes & 100 \\
\hline Queue & Drop Tail \\
\hline Antenna & Omni Directional \\
\hline Initial Energy & $10 \mathrm{~J}$ \\
\hline Area & $600 * 600$ sq. $\mathrm{m}$ \\
\hline
\end{tabular}

The comparison was done on the basis of energy consumption, throughput, packet delivery ratio and number of uncovered nodes.

The energy consumption signifies the network lifetime, as lesser is the energy consumed in the network more is the lifetime and vice-versa.

Packet delivery ratio is the percentage of the packets successfully delivered in the network. Mathematically it is ratio of number of packets received to the number of packets sent in the network.

Throughput is defined as the amount of data received at the base station per unit of time.

The graphs shown below represents the values of these parameters achieved by simulation of the network under existing scheme and the proposed scheme.

\section{GRAPH REPRESENTATION:}




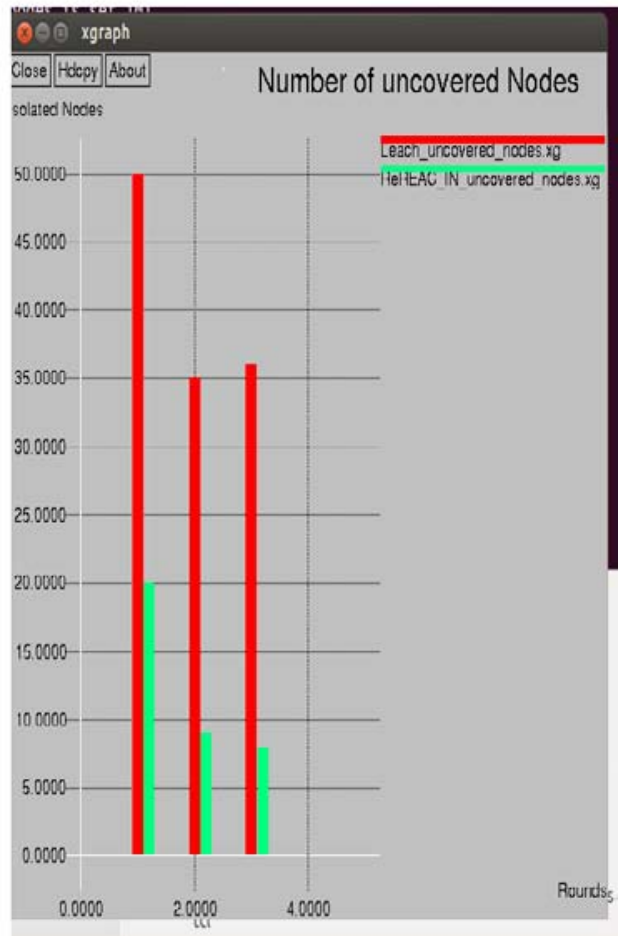

Graph 4 :number of uncovered nodes between LEACH and RE REAC-I

\section{COMPARISON BETWEEN LEACH AND RE REAC-IN}

\begin{tabular}{|c|c|c|}
\hline parameter & LEACH & REREAC -IN \\
\hline Remaining Energy & 3.6166 Joules & 4.52137 Joules \\
\hline Throughput & 4085.76 Kbps & 5142.53 kbps \\
\hline $\begin{array}{c}\text { Packet Delivery } \\
\text { Ratio }\end{array}$ & 0.749692 & 0.909861 \\
\hline $\begin{array}{l}\text { Number of } \\
\text { uncovered nodes }\end{array}$ & $50,35,36$ & $20,9,8$ \\
\hline
\end{tabular}

\section{CONCLUSION:}

\begin{tabular}{|l|l|l|}
\hline Leach & REAC-IN & RE REAC-IN \\
\hline $\begin{array}{l}\text { Random head } \\
\text { selection }\end{array}$ & $\begin{array}{l}\text { Selection } \\
\text { According to } \\
\text { regional energy }\end{array}$ & $\begin{array}{l}\text { Selection } \\
\text { according to } \\
\text { regional energy }\end{array}$ \\
\hline $\begin{array}{l}\text { Isolated nodes not } \\
\text { considered for } \\
\text { connectivity }\end{array}$ & $\begin{array}{l}\text { Isolated nodes } \\
\text { already } \\
\text { connected to } \\
\text { nearest cluster } \\
\text { head }\end{array}$ & $\begin{array}{l}\text { Isolated nodes } \\
\text { already } \\
\text { connected to } \\
\text { nearest cluster } \\
\text { head }\end{array}$ \\
\hline
\end{tabular}

\begin{tabular}{|l|l|l|}
\hline $\begin{array}{l}\text { Directly } \\
\text { communicate with } \\
\text { base station }\end{array}$ & $\begin{array}{l}\text { Multi hop } \\
\text { communication }\end{array}$ & $\begin{array}{l}\text { Multi hop } \\
\text { communication }\end{array}$ \\
\hline Low delivery rate & $\begin{array}{l}\text { High delivery } \\
\text { rate }\end{array}$ & $\begin{array}{l}\text { High delivery } \\
\text { rate }\end{array}$ \\
\hline Low throughput & $\begin{array}{l}\text { High } \\
\text { throughput }\end{array}$ & High throughput \\
\hline Moderately efficient & Highly efficient & Highly efficient \\
\hline
\end{tabular}

The comparison was done on the basis of four parameters; all these showed an improvement over the existing scheme. graph 4: shows that in Re Reac-IN number of unconnected nodes has been less. Thus it can be fairly concluded that more and more number of nodes have been provided connectivity to forward the data. This eventually reduces their communication distance with the base station. This results in lesser energy consumption in the network. Also, over a shorter range communication the packets are transmitted correctly, thus leading to improvement in packet delivery ratio as well as throughput of the network.

In future, this scheme can be analyzed against various security attacks that wireless sensor network is prone to.

\section{REFERENCES}

[1]. Mandeep sidhu and Manpreet Singh “optimizing the clustering process using relay nodes in wsn “, 5th international conference on advancements in engineering and technology; paper number 79 ; MARCH 2017.

[2] Praveen Chaturvedi , "Introduction to Wireless Sensor Networks" in International Journal of Advanced Research in Computer Science and Software Engineering volume 2 ; issue 10, October 2012.

[3] R. Logambigai, S. Ganapathy, A. Kannan,” Cluster Based Routing with Isolated Nodes in WSN" in International Journal for Research in Applied Science \& Engineering Technology (IJRASET) ; volume 4 ;issue 3 ; March 2016.

[4] .Jenq-Shiou Leu, Tung-Hung Chiang, MinChieh Yu, and Kuan-Wu Su. "Energy Efficient Clustering Scheme for Prolonging the Lifetime of Wireless Sensor Network with Isolated Nodes". IEEE Communications Letters ; volume 19 ; issue :2 ; feb 2015.

[5] M.Natarajan R.Arthi Ramanujan, K.Murugan Ramanujan, "Energy Aware Optimal Cluster Head Selection in Wireless Sensor Networks" IEEE - computing ,communication and network technologies (ICCCNT), $4^{\mathrm{TH}}$ INTERNATIONAL CONFERENCE , July 2013.

[6] "A comparative study on leach protocols and their variants a study" by heenadhawan and sandeep wariach volume 95,number 8 ,June 2014 .

[7] D. J. Dechene, A. El Jardali, M. Luccini, and A. Sauer." Clustering Algorithms for Wireless Sensor Networks" Ontario London, Ontario, SCIENCE DIRECT ,Canada 2002.

[8] Xia Li, Wang Gang, Liu Zongqi, Zhang Yanyan, "An energy-efficient routing protocol based on particle swarm clustering algorithm and inter-cluster routing algorithm for WSN" in IEEE CONTROL AND DECISION CONFERENCE (CCDC) $201325^{\mathrm{TH}}$ CHINESE.

[9] K Venice and S Deepika, "Effective and secure transmission approach for multi cluster based wireless sensor networks" in international journal of innovative 
research in computer and communication engineering, VOLUME 2, ISSUE 3 MARCH2014.

[10] Agam Gupta and Mansi Gupta, "Improved energy efficiency and reduced delay using self-knowledge with SCHP in wireless sensor networks" in international journal of advanced research in computer science and management studies, VOLUME 2,ISSUE 8 August 2014.

[11] Sk Kajal ArefinImon, Adnan Khan, Mario Di Francesco, and Sajal K. Das. "Energy-Efficient Randomized Switching for Maximizing Lifetime in Tree-Based Wireless Sensor Networks”. IEEE/ACM Transactions On Networking, volume 23,ISSUE 5,October 2015. 